\title{
Triple threat: pancreatic cystic lesion presenting with spontaneous hemorrhage is found to harbor three distinct neoplasms
}

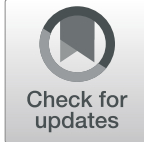

\author{
Da Eun Cha ${ }^{1 *} \mathbb{D}$, Callie Horn ${ }^{1}$ and Michael Passeri ${ }^{2}$
}

\begin{abstract}
Background: Pancreatic serous cystadenoma (SCA) is a benign, cystic lesion with an indolent growth pattern. Complications such as spontaneous hemorrhage or malignant transformation from SCA are extremely rare. Our case report describes an unusual presentation of a patient with a previously diagnosed SCA, made unique by the presence of three separate neoplasms in the final specimen.

Case presentation: A 74-year-old male with a previous diagnosis of SCA presented emergently with epigastric pain and non-bilious vomiting. Laboratory results were notable for a hemoglobin of $8.3 \mathrm{~g} / \mathrm{dl}$. CT scan of the abdomen demonstrated a complex, solid-cystic mass in the pancreatic head with a large hematoma and questionable focus of active hemorrhage. Surgical resection was recommended due to the risk of malignancy, possibility of re-bleeding, and symptoms of severe duodenal compression. Pancreaticoduodenectomy was performed, and final pathology demonstrated three separate neoplasms: serous cystadenoma, intraductal papillary mucinous neoplasm, and neuroendocrine tumor.
\end{abstract}

Conclusion: While pancreatic SCA are benign tumors that can be observed safely in the majority of cases, surgical intervention is often indicated in patients with large, symptomatic cysts or when diagnosis is unclear. When undergoing surveillance, it is crucial for both the patient and the care team to be aware of the possibility of rare, but life-threatening complications, such as hemorrhage. Likewise, the possibility of misdiagnosis or concurrent neoplasia should be considered.

Keywords: Pancreatic cystic neoplasm, Pancreatic serous cystadenoma, Hemorrhage, Intraductal papillary mucinous neoplasm, Pancreatic neuroendocrine tumor

\section{Background}

Pancreatic cystic neoplasm (PCN) represents a diverse group of pathologic entities with a wide range of clinical implications-most commonly, serous cystadenoma (SCA), mucinous cystic neoplasm (MCN), intraductal papillary mucinous neoplasm (IPMN), cystic pancreatic neuroendocrine tumor (pNET), and solid pseudopapillary neoplasm (SPEN) [1]. PCN is detected with greater

\footnotetext{
* Correspondence: Daeun.cha@mountsinai.org

${ }^{1}$ Surgery Department, Mount Sinai West and Morningside, 425 West 59th Street, 7th Floor, New York, NY 10019, USA

Full list of author information is available at the end of the article
}

frequency due to the growing ubiquity and high resolution of cross-sectional imaging techniques and endoscopic ultrasound (EUS). Computed tomography (CT) and magnetic resonance imaging (MRI) examinations suggest $2.5-5 \%$ prevalence of pancreatic cysts with PCN representing $15-20 \%$ of all cysts [1]. Of these, SCA is generally considered benign, only rarely degenerating into serous cystadenocarcinoma ( $<1 \%$ of cases) [2]. MCN and IPMN, by contrast, have greater malignant potential, with an estimated malignancy risk of $10-50 \%$ for $\mathrm{MCN}$ and up to $60 \%$ for IPMN involving the main pancreatic duct [1]. Differentiating between these PCN 
subtypes on cross-sectional imaging is desirable, but the differences may not be clearly discernible based on imaging alone. Determining the optimal surveillance interval and the need for invasive testing and operative resection are often complex clinical decisions, especially when the etiology of the PCN is uncertain.

Observation has been favored historically once SCA is diagnosed, as the malignant potential is extremely low. Asymptomatic patients with SCA usually undergo surveillance with serial imaging [3], and resection is reserved for symptomatic patients with SCA or uncertainty of diagnosis [2]. Larger tumors are more likely to cause symptoms like abdominal pain, nausea, and vomiting due to local compression [4]. In rare cases, giant SCA may cause splenic vein compression leading to gastric varices or obstructive jaundice requiring surgical resection [5, 6]. A spontaneous hemorrhage from SCA is extremely rare, with only a few reported cases in literature [7-9], and as such, the threat of bleeding has not significantly influenced treatment or surveillance algorithms.

We present a rare case of a patient who presented with spontaneous hemorrhage from a large cystic neoplasm in the pancreatic head diagnosed as SCA 5 years earlier. The patient underwent pancreaticoduodenectomy, and the final pathology was remarkable for three separate neoplasms: SCA, IPMN, and pNET. We discuss the risks posed by SCA, as well as the importance of structured surveillance once SCA is suspected.

\section{Case presentation}

A 74-year-old male, with a past medical history of SCA diagnosed 5 years prior to presentation, hypertension, nephrolithiasis, and childhood polio with consequent bilateral lower extremity atrophy, presented to the emergency department with epigastric pain associated with persistent, non-bilious vomiting. Patient mentioned a history of a pancreatic cyst, which he had been told was harmless. A CT scan from 5 years prior to his presentation demonstrated a $10 \times 8 \mathrm{~cm}$ septated cystic lesion in the head of the pancreas (Fig. 1a). In the intervening 5 years, no additional diagnostic work-up or surveillance had been performed.

Upon arrival at the emergency department, the patient was tachypneic, but his blood pressure and heart rate were normal. His abdomen was soft, mildly distended, with generalized abdominal tenderness worse in the upper quadrants bilaterally. Laboratory results were significant for a leukocytosis of $17,000 / \mu \mathrm{L}$, and a hemoglobin of $8.3 \mathrm{~g} / \mathrm{dL}$. A CT scan of the abdomen demonstrated a complex, septated cystic mass in the pancreatic head measuring $10 \mathrm{~cm}$, with a new solid component and an adjacent hematoma measuring $14 \times 6 \mathrm{~cm}$, with apparent focus of active hemorrhage centrally. The
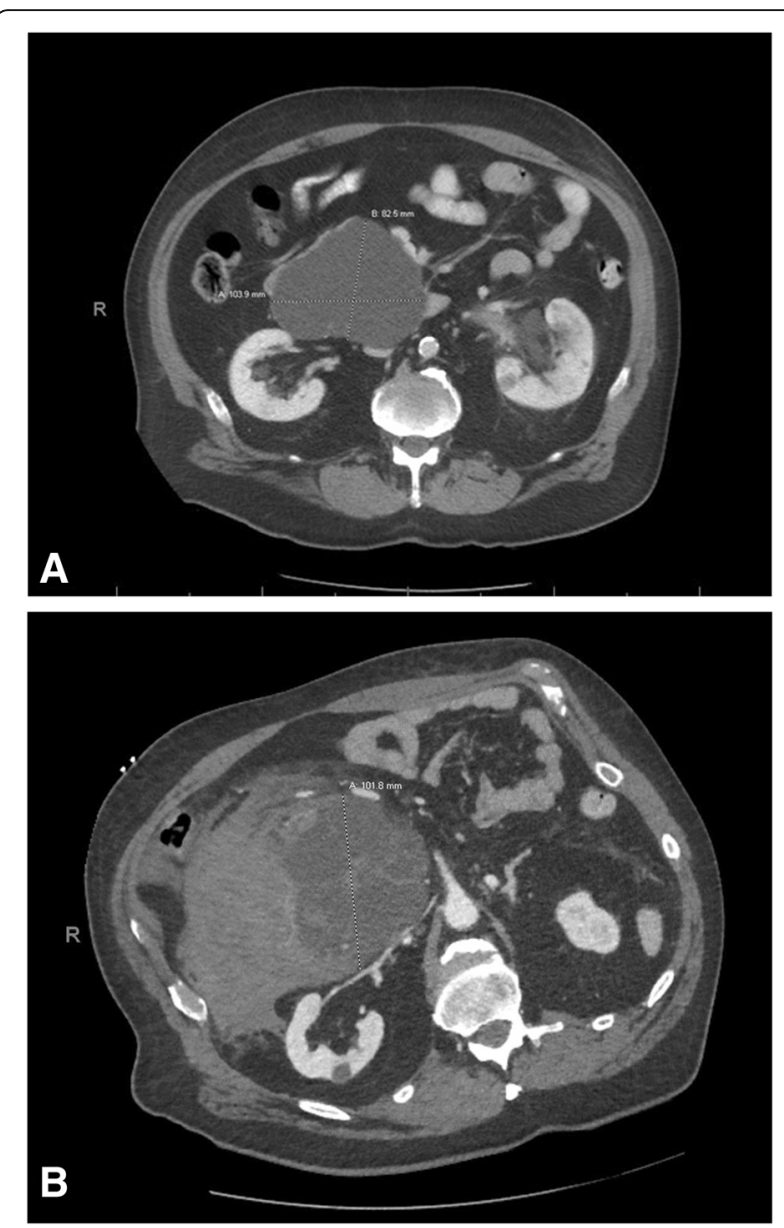

Fig. 1 a Representative image of CT from 2015 demonstrating $10 \times$ $8 \mathrm{~cm}$ septated cystic lesion in the head of pancreas. $\mathbf{b}$ Image of CT from 2020 demonstrating complex, septated cystic mass now measuring $14 \times 6 \mathrm{~cm}$, with focus of active hemorrhage

combined mass-effect of the solid/cystic lesion and the associated hematoma led to a severe compression of the duodenum (Fig. 1b).

Embolization was first considered given the contrast blush seen on CT. However, close interval hemoglobin measurements remained stable and the patient was admitted to the intensive care unit for continued observation.

Several factors and the patient's clinical status were taken into consideration in determining the next steps in management. The large size of the cystic lesion and the new solid component raised concerns for malignant degeneration [10]. Spontaneous hemorrhage from this lesion and the possibility of a recurrent episode in the near future also imposed a sense of urgency. The presence of persistent symptoms from duodenal compression was also concerning. Thus, the combination of the malignant potential, bleeding risk, and duodenal obstruction led to the recommendation for surgical resection. 
The patient was taken to the OR by the hepatopancreatobiliary (HPB) surgery team for a Whipple procedure on hospital day 3. Intraoperatively, a large hematoma was found adjacent to the lesion, extending posterolaterally from the second portion of the duodenum compressing the duodenal lumen. There was no evidence of vascular invasion.

Pathology from the specimen demonstrated chronically inflamed pancreatic tissue and three separate neoplasms. A main duct IMPN (Fig. 2a) was seen arising in the background of macrocystic SCA, which measured 13 $\times 8 \mathrm{~cm}$ (Fig. 2b). The lesion also contained a welldifferentiated neuroendocrine tumor $(0.4 \mathrm{~cm})$ (Fig. 2c), which was synaptophysin and chromogranin positive and with Ki67 < 3\% (Fig. 2d). Pathologists also noted the presence of marked secondary inflammatory and degenerative changes, with prominent necrotizing pancreatitis, fat necrosis, hemorrhage, and fibrosis, accompanying all three lesions. 18 lymph nodes were evaluated, all of which were negative for metastatic disease. The distal pancreatic margin was associated with focally exuberant inflammatory and degenerative changes, including ductal dilation and focal pancreatic intraepithelial neoplasia (PanIN 1).

The postoperative course was uneventful. Patient was discharged on postoperative day 11 , delayed due to the patient's need for extensive physical therapy given preexisting conditions. The patient has since recovered from the procedure and is doing well. Given the absence of invasive carcinoma, no adjuvant therapy was offered. Surveillance imaging is scheduled 6 months postoperatively.

\section{Discussion}

SCAs are benign tumors that exhibit an indolent growth pattern. They are more commonly found in women, with a median age of 58 years at diagnosis [10]. Many are diagnosed incidentally on imaging studies, as up to $61 \%$ of patients are asymptomatic [10]. Risk of malignant transformation to cystadenocarcinoma is reported to be as low as $0.1 \%$ and SCA-specific mortality is reported to be nearly zero [10].

Given the benign natural history of SCA, observation of asymptomatic patients is typically recommended. There is currently no consensus on management strategies, with recommendations ranging from surveillance for asymptomatic patients to surgical resection depending on various factors such as SCA size, location, or growth rates $[1,3,4,10]$. These recommendations hinge on the accuracy of the diagnosis. Distinguishing SCA from other potentially malignant forms of cystic neoplasm with certainty is difficult. One study found that of 2622 cases of resected cystic neoplasm, 60\% underwent surgical resection because of uncertainty in diagnosis [10]. Even with improvements in the resolution of crosssectional imaging modalities, the ability to distinguish

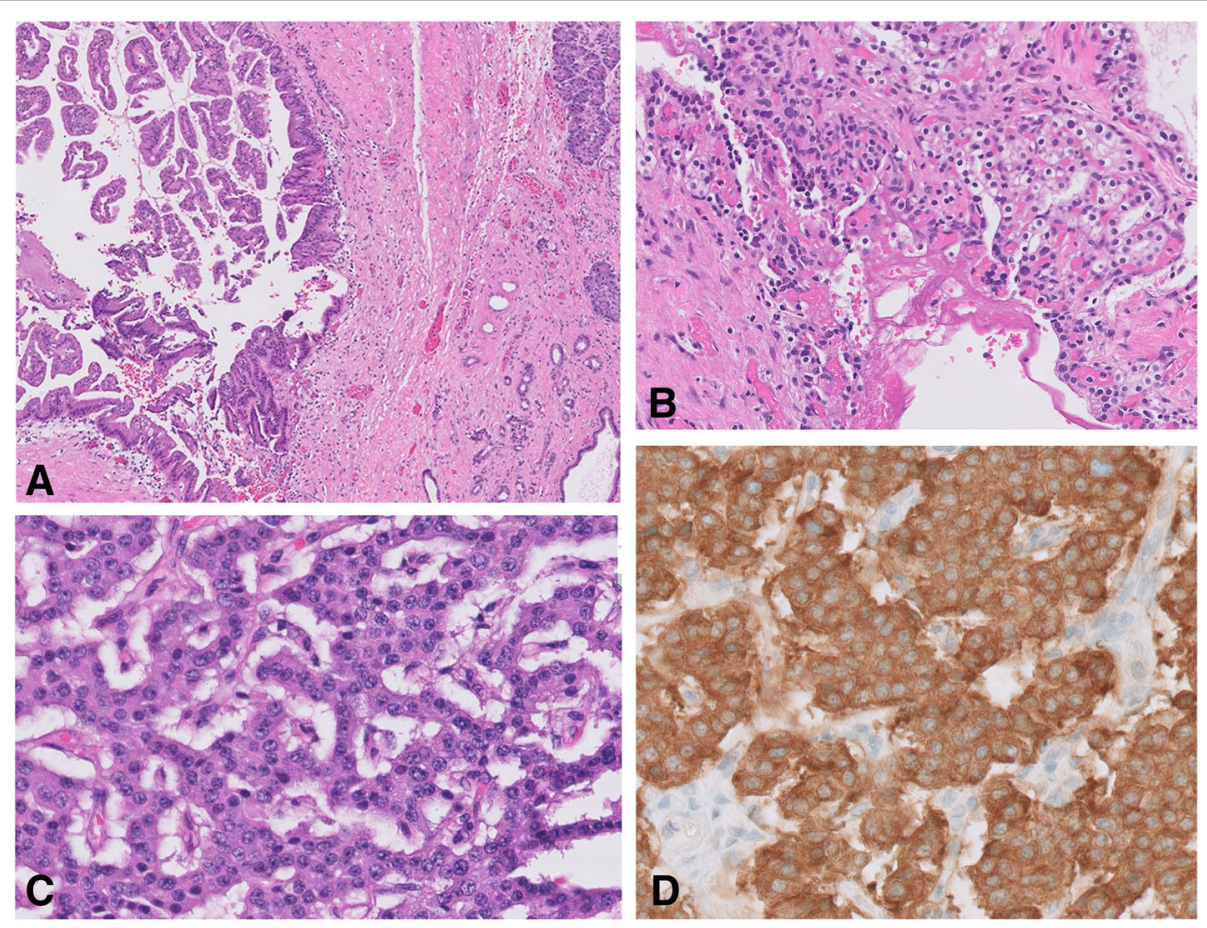

Fig. 2 Histology slides of a intraductal papillary mucinous neoplasm with adjacent non-neoplastic pancreas tissue (5x), H\&E stain, b serous cystadenoma (20x), H\&E stain, c neuroendocrine tumor (40x), H\&E stain, d neuroendocrine tumor (40x), and synaptophysin immuno-stain 
between subtypes of $\mathrm{PCN}$ has been limited: the accuracy rate for identifying the subtype of $\mathrm{PCN}$ is reported to range from 40 to $81 \%$ for CT and 40 to $95 \%$ for MRI [3]. Certain morphologic patterns of SCA add to the complexity of diagnosis. While $70 \%$ of SCA exhibit a microcystic pattern, in which the tumor is composed of numerous, small cysts, some, by contrast, exhibit a macrocystic pattern, which describes fewer large cysts [11]. The macrocystic pattern can resemble MCN or IPMN on imaging [11]. The presence of an intratumoral hemorrhage, as in our patient, also leads to difficulty in differentiation between cystic and solid tumors, since cross-sectional imaging can show heterogeneous enhancement from hemorrhage, necrosis, and cystic degeneration [11].

Endoscopic ultrasound (EUS) can provide supplemental information, especially if fine needle aspiration (FNA) is performed for fluid analysis. Cyst fluid carcinoembryonic antigen (CEA) level has been useful in differentiating between mucinous and non-mucinous pancreatic cysts [12]. Vascular endothelial growth factor (VEGF) has been identified as an accurate SCA biomarker [12]. Carr et al. found that combined CEA and VEGF cyst fluid levels had 95\% sensitivity and $100 \%$ specificity for the diagnosis of SCA. While these results are encouraging, EUS with FNA may not be technically feasible or may not yield sufficient aspirate for analysis for all patients.

Even when a clear diagnosis of SCA is made based on radiographic and clinical findings, different management strategies have been recommended. The European Study Group on cystic tumors of the pancreas recommends observation for 1 year for asymptomatic patients followed by symptom-based follow-up and surgical resection for patients with symptoms related to compression of adjacent organs [3]. Another single center study on SCA recommends offering surgical resection for symptomatic patients and for patients with SCA measuring $\geq 4 \mathrm{~cm}$, as larger tumors are more likely to become symptomatic and to be associated with faster growth rates [4]. Invasive diagnostic modalities, even resection, are indicated when different types of cystic neoplasm, especially those harboring malignant potential such as MCN or IPMN, cannot be confidently excluded [10].

Our case report describes a patient who developed spontaneous hemorrhage associated with previously diagnosed SCA. Only a few cases of hemorrhage related to SCA have been reported based on our literature search. In two case reports, patients presented with acute abdomen from hemorrhage and underwent distal pancreatectomy of large cysts with a diameter $>10 \mathrm{~cm}[7,8]$. While rare, hemorrhage associated with SCA may be lifethreatening and requires prompt identification and surgical intervention.
Our case report is also unique because the final pathology remarkably identified three different types of neoplasm: macrocytic serous cystadenoma, main duct IPMN, and well-differentiated neuroendocrine tumor. SCAs have been found concurrently with one other type of pancreatic tumors within the same excised specimen, such as IPMN, pancreatic ductal adenocarcinomas, neuroendocrine tumors, and metastatic tumors. In a study of 193 pancreatic serous neoplasms, concurrent neoplasms were found in 13\% (27) of cases [13]. Most commonly identified synchronous neoplasms were pancreatic neuroendocrine tumors $(6 \%, 12 / 193)$ and IPMN $(<1 \%, 1 / 193)$ [13]. Incidental PanIN was found in 83 cases [13]. Based on our literature search, no prior cases of SCA with two different types of neoplasms have been reported. The possibility of SCA harboring other neoplasms with malignant potential should be considered in outlining a management plan.

\section{Conclusion}

We report the first case of SCA with two synchronous pancreatic neoplasms. While pancreatic SCA are typically benign tumors, the possibility of misdiagnosis or concurrent neoplasia should be considered. When opting for observation, it is crucial for both the patient and the care team to be aware that the tumor may grow, causing local mass effect and eventual symptoms, and that lifethreatening complications may arise in rare cases.

\section{Abbreviations \\ SCA: Serous cystadenoma; PCN: Pancreatic cystic neoplasm; CT: Computed tomography; MRI: Magnetic resonance image; MCN: Mucinous cystic neoplasm; SPEN: Solid pseudopapillary neoplasm; \\ HPB: Hepatopancreatobiliary; FNA: Fine needle aspiration; CEA: Carcinoembryonic antigen; VEGF: Vascular endothelial growth factor; PanIN: Pancreatic intraepithelial neoplasia}

\section{Acknowledgements}

None

Authors' contributions

DC: writing of the manuscript. $\mathrm{CH}$ : writing of the manuscript. MP: writing and editing of the manuscript. The authors read and approved the final manuscript.

Funding

This research did not receive any specific grants or funding.

Availability of data and materials

There is no dataset as this is a case report. Data/details of the patient available upon request.

Ethics approval and consent to participate

Ethics committee approval was not required for this case as this is a case report.

\section{Consent for publication}

Informed consent for publication of clinical data/details/images was obtained from patient. A copy of consent is available for review by the Editor of this journal. 


\section{Competing interests}

There were no competing interests in completing this case report.

\section{Author details}

'Surgery Department, Mount Sinai West and Morningside, 425 West 59th Street, 7th Floor, New York, NY 10019, USA. ${ }^{2}$ HPB Surgery and Surgical Oncology, Valley Medical Group, Luckow Plaza, One Valley Health Plaza, Paramus, NJ 07652, USA.

Received: 28 August 2020 Accepted: 2 January 2021

Published online: 15 January 2021

\section{References}

1. Chandwani R, Allen PJ. Cystic neoplasms of the pancreas. Annu Rev Med. 2016;67:45-57.

2. Zhang XP, Yu ZX, Zhao YP, Dai MH. Current perspectives on pancreatic serous cystic neoplasms: diagnosis, management and beyond. World J Gastrointest Surg. 2016;8(3):202-11. https://doi.org/10.4240/wjgs.v8.i3.202.

3. The European study group on cystic tumours of the pancreas. European evidence-based guidelines on pancreatic cystic neoplasms. Gut. 2018;67: 789-804. https://doi.org/10.1136/gutjil-2018-316027.

4. Tseng JF, Warshaw AL, Sahani DV, Lauwers GY, Rattner DW, Fernandez-del CC. Serous cystadenoma of the pancreas tumor growth rates and recommendations for treatment. Ann Surg. 2005;242:413-21.

5. Pointer L, Rothermel LD, Strosberg C, Anaya D, Hodul P. Giant symptomatic serous cystadenoma mimicking carcinoma: a case report and literature review. Int J Surg Case Rep. 2019;60:106-10.

6. Gilmore RC, Kalmar CL, He J. Serous cystadenoma of the pancreas: rare presentation of obstructive jaundice. Am Surg. 2020;00:1-3. https://doi.org/ 10.1177/0003134820923315.

7. Amaral JA, Serodio M, Ramalhosa F, Tralhao JG. Pancreatic microcystic serous cystadenoma a lethal disease? Rare case of life-threatening haemorrhage. BMJ Case Rep. 2020;13:e233562. https://doi.org/10.1136/bcr2019-233562.

8. Ashkzaran H, Coenegrachts K, Steyaert L, Vandelanotte M, van den Berghe I, Verstraete $K$, et al. An unusual presentation of pancreatic serous cystadenoma with acute hemorrhage. JBR BTR. 2007;90:44-6.

9. Tamura S, Yamamoto Y, Okamura Y, Sugiura T, Ito T, Ashida R, et al. A case of duodenal hemorrhage due to arteriovenous malformation around a serous cystic neoplasm. Surg Case Rep. 2018;4:140.

10. Jais B, Rebours V, Malleo G, Salvia R, Fontana M, Maggino L, et al. Serous cystic neoplasm of the pancreas: a multinational study of 2622 patients under the auspices of the international association of pancreatology and European pancreatic club (European study group on cystic tumors of the pancreas). Gut. 2016;65:305-12. https://doi.org/10.1136/gutjnl-2015-309638.

11. Choi JY, Kim MJ, Lee JY, Lim JS, Chung JJ, Kim KW, et al. Typical and atypical manifestations of serous cystadenoma of the pancreas: imaging findings with pathologic correlation. AJR. 2009;193:136-42.

12. Carr RA, Yip-Schneider MT, Dolejs S, Hancock BA, Wu H, Radovich M, et al. Pancreatic cyst fluid vascular endothelial growth factor $A$ and carcinoembryonic antigen: a highly accurate test for the diagnosis of serous cystic neoplasm. J Am Coll Surg. 2017;225:93-100.

13. Reid MD, Choi HJ, Memis B, Krasinskas AM, Jang KT, Akkas G, et al. Serous neoplasm of the pancreas a clinicopathologic analysis of 193 cases and literature review with new insights on macrocystic and solid variants and critical reappraisal of so-called "serous cystadenocarcinoma". Am J Surg Pathol. 2015;39:1597-610.

\section{Publisher's Note}

Springer Nature remains neutral with regard to jurisdictional claims in published maps and institutional affiliations.

Ready to submit your research? Choose BMC and benefit from:

- fast, convenient online submission

- thorough peer review by experienced researchers in your field

- rapid publication on acceptance

- support for research data, including large and complex data types

- gold Open Access which fosters wider collaboration and increased citations

- maximum visibility for your research: over $100 \mathrm{M}$ website views per year

At BMC, research is always in progress.

Learn more biomedcentral.com/submissions 